\title{
Degree of Achievability of Omnidirectional Motion in Various Mobile Robot Designs: A Review
}

\author{
Ajju R. Justus \\ Department of Mechanical Engineering Sree Chitra Thirunal College of Engineering
}

\begin{tabular}{l}
\hline \hline Article Info \\
\hline Article history: \\
Received Nov 15, 2015 \\
Revised Jan 25, 2016 \\
Accepted Feb 10, 2016 \\
\hline
\end{tabular}

\section{Keyword:}

Driving Method

Omnidirectional

Spherical Mobile Robot

\begin{abstract}
In the current field of robotics, many new robots are being developed based on different working principles. Each robot has its own strengths and weaknesses. Omnidirectional mobility is a major functional requirement for performing more complex actions. This article is a comprehensive study of some of the robots developed by different people with distinctive driving mechanisms. The actuation, driving method, operating conditions and control mechanism of all the selected robots is studied. Then finally, all the considered robots are compared based on various parameters to gauge the efficiency and degree of achievability of omnidirectional motion in each robot. Therefore, by the end of this article, we can have an understanding of how much effective each driving mechanism is in producing omnidirectional mobility.
\end{abstract}

Copyright () 2016 Institute of Advanced Engineering and Science. All rights reserved.

\section{Corresponding Author:}

Ajju R. Justus,

Department of Mechanical Engineering,

Sree Chitra Thirunal College of Engineering,

Pappanamcode, Thiruvananthapuram, Kerala 695018 Mob no: +91-9633702955

E mail: ajurjustus@yahoo.co.in

\section{INTRODUCTION}

A robot can be defined as any automated machine which is able to replace human effort, which need not resemble humans in appearances or perform functions in a humanlike manner [1]. Robots or automatic machines had its beginning from ancient mythologies. Robotics is now, a fast-developing field of study with massive and innovative technological breakthroughs.

Early robots used to move around by means of wheeled or legged locomotion. However with the increase in complexity of functions of the robots, operational spaces got more restricted and complicated. It required the robots to be highly maneuverable. New dynamic and robust types of locomotion were needed to be developed. The existing wheeled and legged locomotion were found to be inadequate for performing more complex tasks. Thus, many new driving methods were developed, which would allow the robot to move Omni directionally. Omni directional motion can be defined as the ability of the system to move in any direction at any instant of time from any configuration. Conventional wheels as in automobiles are able to move merely in the forward or backward directions and rotation about its center of gravity with a minimum radius of turning. It means that suppose we want to move in a direction parallel to the axis of the wheel, we can achieve that only by a series of complex maneuvers resulting in the reorientation of the wheel in the desired direction. Since they can't move in every direction at a particular instant, they are not omnidirectional. Over the years, many different classes of omnidirectional robots have been developed based on distinctive operating principles. A spherical robot which had an active wheel inside the sphere to generate planar motion was developed by A.Halme et al [2]. Another design was developed by J Dias et al in which a four-wheeled vehicle was kept inside the sphere. The vehicle was driven to make the external shell move in the required direction [3]. 
Another design drives a ball which is made to impart motion to the external shell [4]. Non spherical mobile are also developed like the Uranus robot which makes use of Mecanum wheels to achieve omnidirectional mobility [5]. Many such robots were designed, which are discussed in the coming sections.

\section{SOME DISTINCTIVE MOBILE ROBOTS}

Any robot capable of locomotion can be described as a mobile robot. Under mobile robots, only those robots which are capable of exhibiting omnidirectional motion are studied. Under this criterion, most of the omnidirectional robots considered fall under the category of spherical mobile robots. Spherical robots refer to those robots whose external structure is in the form of a sphere. The morphology of the robots provides additional advantages over other kinds of robots like fast collision recovery, symmetric geometry, which helps in achieving omnidirectional motion without reorienting itself. So its motion is holonomic in nature. The driving mechanism for these robots is typically enclosed within the spherical shell. A robotic drive is said to be Holonomic if the total degrees of freedom are equal to the controllable degrees of freedom of the robot. If the total degrees of freedom is more than the controllable degrees of freedom, it is known as a Non Holonomic drive. If the total degrees of freedom of a robot are less than the controllable degrees of freedom, it is known as a Redundant drive [6].

Several mobile robots have been developed based on various driving mechanisms. Driving principles based on which almost all mobile robots are developed is given below:

[1] Direct driving method: The torque produced by the motor is directly transmitted to the outer shell as the propelling power for the robot.

[2] Barycenter offset or Gravity method: The barycenter is the center of mass of two revolving bodies. By changing the mass distribution, we can change the barycenter of the robot thereby making it move to attain a new state of equilibrium.

[3] Shell transformation method: The transformation of the outer shell of the robot is being relied upon for its movement.

[4] Angular momentum method: In this method, Control Moment Gyroscopes are added to the spherical robot. The laws of conservation of angular momentum are used to control the movement of the robot. The output toque is proportionally related to the angular momentum of control moment gyroscopes.

Under mobile robots, we have considered about eleven different robots to summarize their driving principles, performance and whether they are able to achieve omnidirectional motion.

\subsection{Ball Robot}

This is probably the first spherical mobile to be developed. The driving mechanism for this robot falls under the category of gravity method [2]. Its working is simple. It consists of a drive unit (wheel and other inside components) placed inside the sphere which is moved to make the outer shell move in the required direction. There are two motors, which are used to drive and steer the driving wheel inside the shell independently. This robot requires two inputs for its locomotion. This robot does not have any kind of feedback mechanism to correct its motion. Therefore it can be defined as an open-loop system [2].

It is difficult to balance the robot as its stability depends on the position of its Inside Drive Unit (IDU) which is hard to assess. Performance of this robot is not available in the literature.

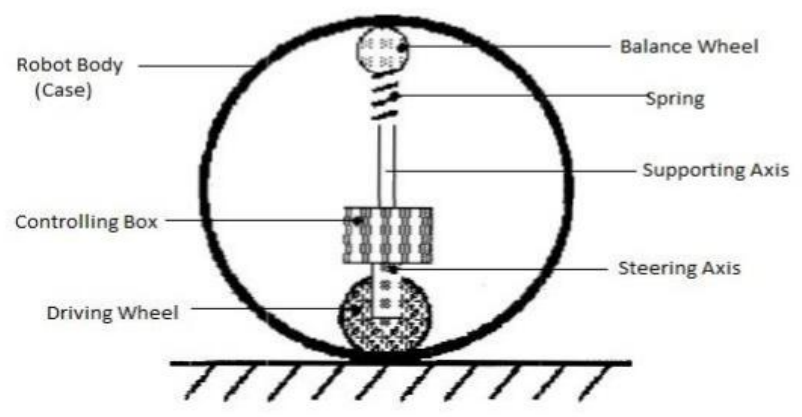

Figure 1. Basic Structure Of The Robot [2]. 
The driving torque of the robot is given by:

$1 * \mathrm{~m}=\mathrm{r}^{*} \mathrm{~m} \operatorname{Sin} \theta$ where

$r$ - Distance between the center of the ball lo the mass center of the Internal Drive Unit,

$\mathrm{m}$ - Mass of IDU,

1 - Distance between the mass center of IDU, and the supporting point of the ball,

$\theta$-inclination angle of IDU

Being the first of its kind, it has various shortcomings like no proper controlling mechanism, not being able to achieve omnidirectional mobility. 2

\subsection{Ball Hamster Design Robot}

This is another kind of spherical robot in which a four-wheeled vehicle is kept inside the shell and allowed to move making the outer shell also move. The four wheels can be controlled independently. Therefore, it is more maneuverable and can generate different motion curves. The position of the center of gravity of the internal unit determines the movement of the robot. The robot is steered by changing the lateral position of the center of mass. Therefore, we can categorize the working of this robot under gravity method [7]. It has a closed-loop control. The feedback of this robot system is achieved by inertial sensing. Even though the wheels act as a differential drive allowing the robot to turn in place, it faces many problems like slippage of the internal unit, Low accuracy in achieving the required motion path etc. The robot is very effective on straight paths or on paths with small curvature [3]. However, it becomes unstable on paths with a bigger curvature. Even though this robot can turn in place, it requires time to move the robot in the required direction. Therefore, this robot cannot claim to achieve omnidirectional mobility. No details of its performance are given in the literature.

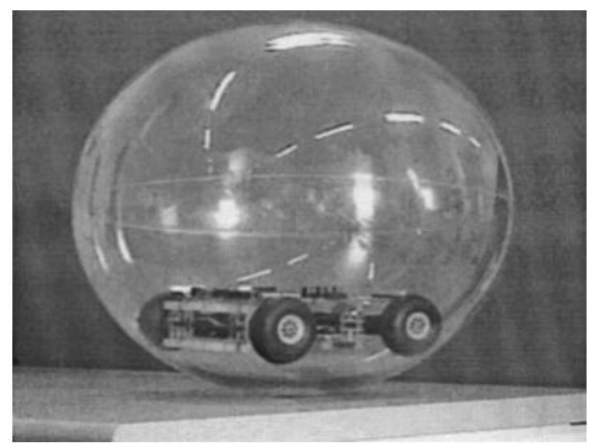

Figure 2. Ball Hamster Prototype Design [3]

\subsection{Uranus}

Mecanum wheels are almost similar in construction to that of universal wheels. Mecanum has a central hub which consists of free moving rollers. But the difference from the universal wheel is in the fact that the rollers are oriented at $45^{\circ}$ to the circumference of the hub [5].

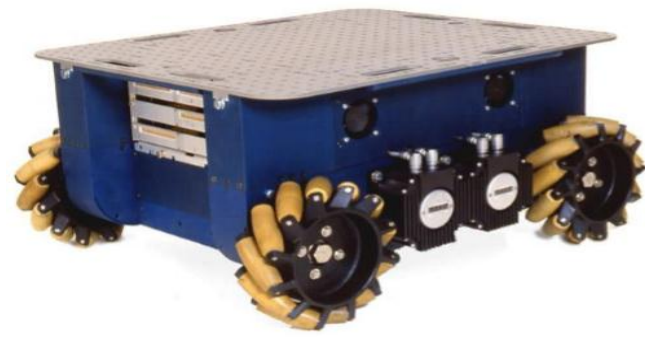

Figure 3. Uranus [8] 
A typical robot utilizing Mecanum wheel is the Uranus robot. It was built by the Mobile Robot Lab of the Carnegie-Mellon University. The external morphology of the robot is rectangular with $75 \mathrm{~cm}$ length, 60 $\mathrm{cm}$ breadth and $40 \mathrm{~cm}$ height. Its weight is about 50kg [9]. The Uranus utilizes direct driving method. It consists of four Mecanum wheels. The wheels are placed in such a way that the rollers on each wheel are opposite in direction to its adjacent wheels. When all the wheels are driven in the same direction, the roller motion of the oppositely oriented wheels cancels each other and the robot moves in forward or backward direction. When the wheels with opposite roller orientations are driven in opposite directions, the rollers act as a screw and therefore, the wheels move sideways. It must be noted that it has four motors to independently transmit the driving force to each wheel. The number of actuators is more than the required degrees of freedom for achieving omnidirectional motion. Therefore, it can lead to redundant actuation. But it can be controlled without much difficulty.

Uranus uses a suspension system which has the advantages of making the ride smoother and Helping all the wheels to continuously have ground contact. The voltage input to Uranus is $24 \mathrm{~V}$ DC. It utilizes a servo controller to properly receive the motion commands and actuate the motors. A maximum torque of $4.7 \mathrm{~nm}$ and thrust of $150 \mathrm{lbs}$ can be generated. It has holonomic capability. The maximum speed that can be achieved by Uranus is almost equal to $1 \mathrm{~m} / \mathrm{s}$ or $3 \mathrm{~km} / \mathrm{hr}$. It was built as a general purpose robot for help research in robot navigation indoors [10].

\subsection{Three-Dimensional Flywheel Robotic System}

This robot belongs to a new breed of spherical robots. Its structure is similar to that of a gimbal. This robot consists of three spherical shells, the outer shell, middle shell, inner shell and a platform connected to the inner shell. The rotation axes of the three shells are defined as $\alpha$-axis, $\beta$ - axis and $\gamma$ - axis. They are orthogonal to each other. In all, there are three actuators to drive the robot. A-axis actuator, $\beta$ - axis actuator and $\gamma$ - axis actuator. A-axis actuator is connected to the outer shell. It produces translator motion to the robot (forward/ backward). The $\beta$ - axis actuator causes rotation of the middle shell, which in turn makes the outer shell to turn and roll about a point by virtue of conservation of angular momentum. In short, we can say that the steering motion of the robot is provided by $\beta$ - axis actuator. $\Gamma$-axis actuator provides balance to the robot. This robot provides improved trajectory control as a result of its closed loop feedback mechanism. The robot is controlled based on the kinematics and dynamics of the system. The total mass of the robot is a little over $325 \mathrm{~kg}$ [11]. It has an outer diameter of about $2 \mathrm{~m}$.

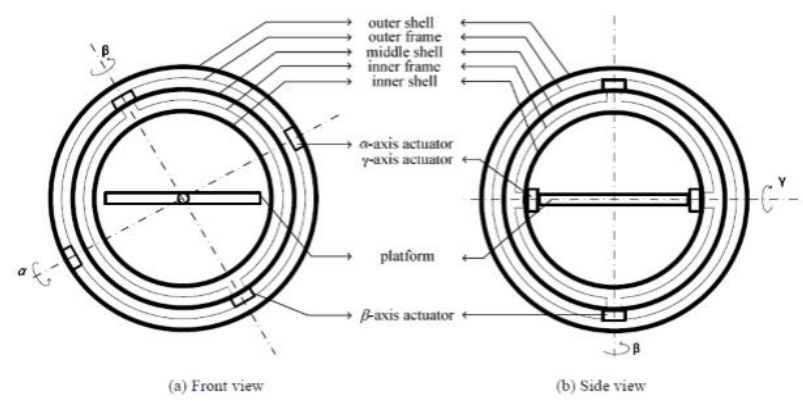

Figure 4. Structure of the robot [11]

The driving method of this robot is a combination of direct driving and conservation of angular momentum. It uses direct driving method for achieving linear motion whereas for steering motion, it goes for conservation of angular momentum method. The three shells of the robot act like three flywheels thereby helping to store rotational energy. It helps in saving actuation energy as the stored energy in the middle and inner shells can be utilized to turn the robot. In spite of its shortcomings like excessive vibrations, this robot has great scope for future applications.

\subsection{Groundbot}

GroundBot is a mobile robot developed by Rotundus. It is a near spherical mobile robot with a high degree of maneuverability. Its weight is about $25 \mathrm{~kg}$ and can navigate through any surface easily. It is slightly larger than a conventional car tire with a diameter or height of about $60 \mathrm{~cm}$. It has a width of about $80 \mathrm{~cm}$. The density of GroundBot is so low that it can even float. GroundBot can run in most terrains at up to 10 $\mathrm{km} / \mathrm{h}(6 \mathrm{mph})$. 


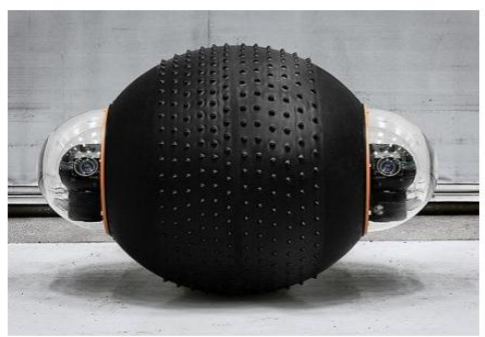

Figure 5. GroundBot [12]

The shell is basically made of polycarbonate over which a high friction coating is given to increase traction forces. The driving mechanism of GroundBot consists of a pendulum which is controlled to move the robot in the desired direction. It comes under gravity method. It utilizes two motors to move the robot in the desired direction [13]. The center of gravity always lies inside the spherical shell. At rest, the center of gravity is close to the ground. So the robot does not tend to tip over, and stability is increased [14].

It has an in -built motor, which moves the pendulum. As the pendulum moves, momentum is developed. Thus, the center of gravity of the GroundBot changes. As a result, it moves in the direction of motion of the pendulum. It has a quick and efficient acceleration and deceleration. GroundBot turns by moving the pendulum to the side. GPS and dead-reckoning are both used to ensure high route accuracy. Since it has a small turning radius for steering movements, it cannot be considered as an omnidirectional robot [7].Li-ion batteries are being used in GroundBot as the power source. GroundBot is widely used in Airports, Security establishments for surveillance purposes.

\subsection{Glory}

Glory is a mobile robot with a spherical outer shell. The external morphology of the robot is spherical. It has the ability to exhibit omnidirectional motion with holonomic capability. The driving mechanism for glory is a bit different and interesting when compared to most other spherical mobile robots. It can be classified under the gravity method. This robot has a total of four spokes, moving radially outwards from the center to the inner circumference of the sphere. The spokes are placed at some specific angles to each other. Weights are placed at each of the four spokes, which can be moved along the spokes using a stepper motor. This total mechanism can be termed as Fixed Internal Propulsion.

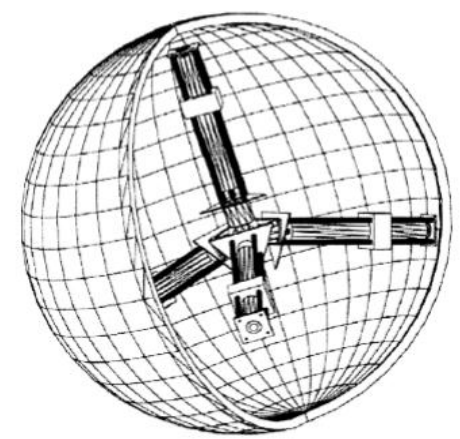

Figure 6. Structure of Glory [15]

Glory is assembled in such a way that it has geometrical symmetry. As a result of this symmetry, the center of mass of the robot, even after including the weights always lie at the geometric center of the robot. The advantage of this is that the robot has increased stability. It does not tend to tip over. Some technical detail of this robot is given by [16].

[1] The angle between the spokes are $109.47^{\circ}$

[2] Mass of each weight is $1.125 \mathrm{~kg}$

[3] Four stepper motor are required

[4] The stepper motor has 200 steps per revolution.

The motion of the stepper motor circuit is controlled by a micro controller. The feedback control of this robot is based on open loop control. Therefore, the scope for inaccurate movements of the robot is quite

Degree of Achievability of Omnidirectional Motion in Various Mobile Robot Designs ... (Ajju R. Justus) 
high. One thing to note is that the motion of this robot is planned and controlled by means of a simple algorithmic iteration and can be easily implemented. It might be a bit slow when we compare its response time with other spherical robots, but it can be neglected. The inaccuracies for the movement of the robot may be due to factors like surface imperfections, center of mass not being at the geometrical center of the robot, open loop control of the robot etc.

\subsection{Omni Crawler Mechanism With Circular Section}

The Omni crawler looks similar to a tank except that is able to move omni directionally. Its locomotion structure is in the form of a capsule. The capability of the crawler to move omni directionally is due to the development of a novel omni ball designed which is used in the omni crawler. The omni ball is used to create the omni crawler. Omni crawler utilizes direct driving method.

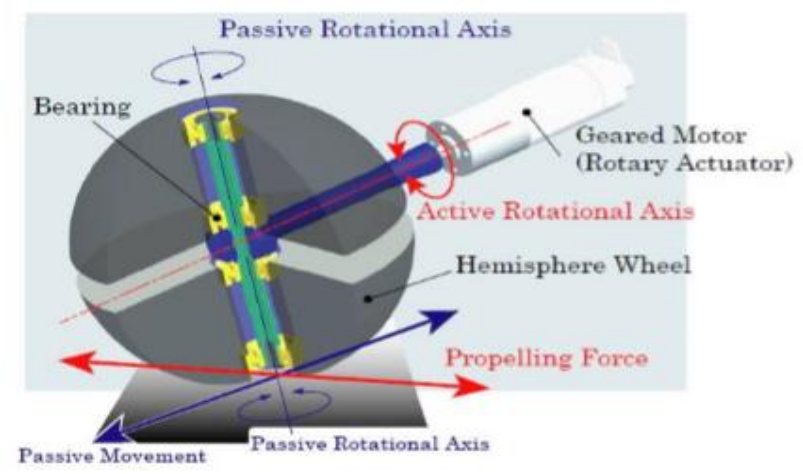

Figure 7. Working principle of Omni ball [17]

The omni ball consists of two hemispheres on either sides of an axle. The two structures can rotate independently or together as a sphere. The active rotation axle is rotated using a roller.

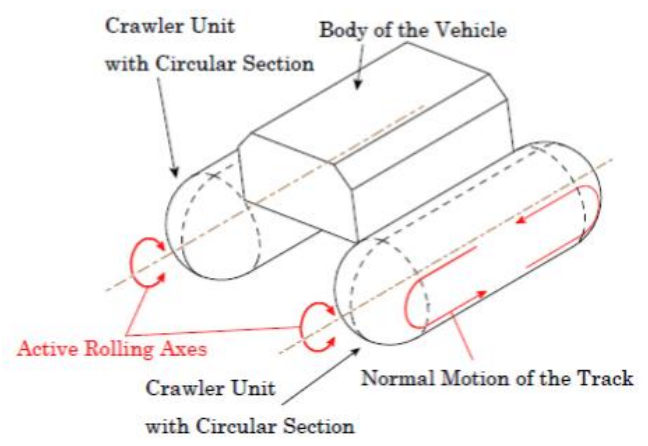

Figure 8. Omni Crawler Prototype Design [17]

The sideways motion of the robot can be achieved by the rotation of the active rotation axis. A total of three motors are required to give the required inputs. A gear motor is used to rotate the inner shaft and gear which in turn rotates the sprockets situated at the ends. This results in the motion in forward or backward direction. Therefore, two motors are used to rotate the left and right crawler units independently for achieving the complete translational motion of the crawler. They have a power rating of $90 \mathrm{~W}$. A single motor is used to rotate both the crawler about their active rolling axes to achieve sideling motion. It has a power rating of 26W. Consequently, the translational and rotational motion can be effectively combined to develop the required output motion. The omni crawler vehicle weights about $8.59 \mathrm{~kg}$ and has a length of $420 \mathrm{~mm}$, width of $314 \mathrm{~mm}$ and height of $190 \mathrm{~mm}$ [17]. 
This robot is very efficient in various operating conditions. It can transverse along the inside and outside surface of pipes. As a result, it can be used as a pipe inspection robot. It also has step climbing ability.

\subsection{Omnicron}

Omnicron is a spherical mobile robot capable of achieving omnidirectional motion with holonomic capability. Omnicron varies from OmniQiu in the sense that the driven ball of the OmniQiu is eliminated and in its place, three omnidirectional wheels are used to drive the spherical outer shell.

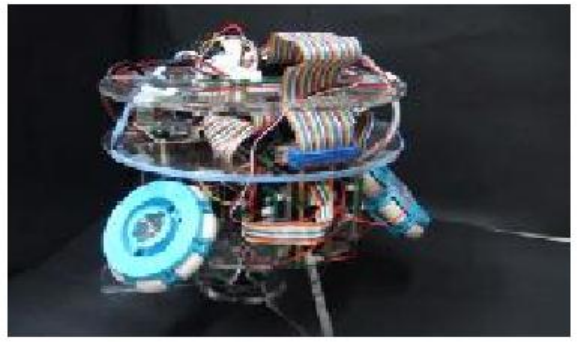

Figure 9. Omnicron Internal driving system [18]

This robot operates under the direct-driving method. Three 90-degree omnidirectional wheels are placed such that their rolling directions are along the three sides of an equilateral triangle. Small passive rollers are present along the circumference of the omnidirectional wheels. So, while the wheels move actively in their rolling directions, they do not act as a constraint to the lateral direction motion. Thus, 3 DOF omnidirectional motion can be achieved by the use of the above said driving mechanism. The feedback is a closed-loop control. Omnicron requires three motors to independently drive the three omnidirectional wheels.

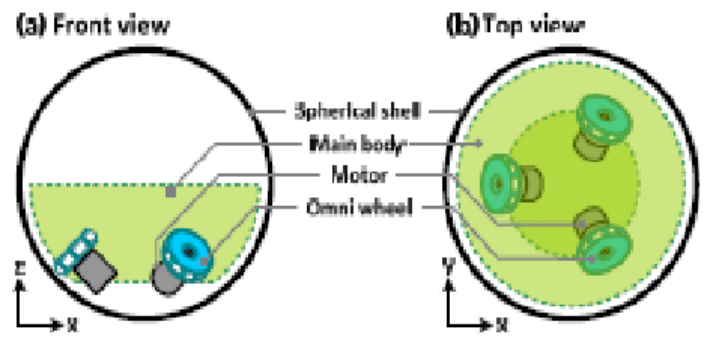

Figure 10. Omnicron structure [18]

The motors are controlled by a real time embedded control system. In short, it utilizes real-time feedback control mechanism. The accuracy of the performance of the Omnicron is not so encouraging from the results of the experimental tests conducted. It has a $10 \%$ error while moving in a straight line. Suppose it has to move forward by a distance of $2 \mathrm{~m}$; it will move ahead by about $2.4 \mathrm{~m}$. On a circular path, it registers a $50 \%$ error [18]. But we should understand that this robot is still in its developing stages and its accuracy can be increased over time by increasing the precision of the driving mechanism.

\subsection{Gyrover I}

Stability of a mobile robot can be broadly classified into two; quasi-static equilibrium and dynamic equilibrium. Quasi-static equilibrium refers to the state of equilibrium at static or near static motions so that the inertial effects on the body can be ignored. Only gravitational forces are considered. Early mobile robots were based on static equilibrium state. The downside is that it cannot be used when dynamic effects on the body have to be considered. As the mobile robots become more complex in terms of their performance and locomotion requirements, dynamic forces and disturbances come into play, which cannot be neglected. For example, a mobile robot required to move at high speeds. In such cases, it is best to consider dynamic equilibrium, which takes into account, the dynamic effects on the body and can use conservation of angular momentum for enhancing the mobility of robot [19]. 
Gyrover is a mobile robot whose locomotion comes under dynamic equilibrium. It uses conservation of angular momentum as its driving principle.

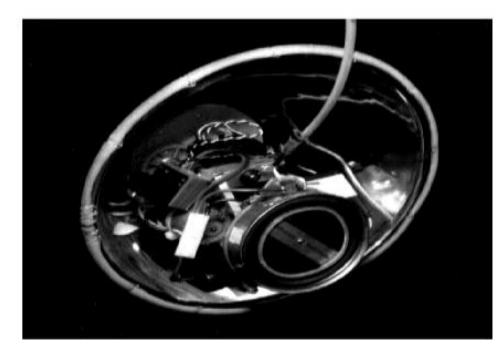

Figure 11. Gyrover I prototype [21]

Gyrover I is a novel, mobile developed from Carnegie Mellon University. It is capable of making sharp turns. Its external morphology is that of a wheel tapered at its circumference. It is novel in the sense that it is stabilized by means of gyroscopic precession. It has a total of three actuators. One motor is used to spin an internal flywheel at a high speed. It is done to provide lateral stability when the Gyrover I is moving slowly or is at rest. Another motor is used to control the steering motions of the Gyrover I and again another motor is used to drive the wheel in the forward or backward direction [19].

A wheel rolling down a slope does not fall over. It is stabilized. Gyrover I works pretty much in the same way. When the Gyrover I leans towards any side, due to the torque induced by the gravitational forces, it precesses at $90^{\circ}$ to the torque applied and so it steers in the direction of inclination of the Gyrover I. Gyrover I has many advantages like the driving mechanism being completely enclosed inside the wheel so as to protect it from dirt and external shocks, ability to turn in place and point contact which results in low resistance to its motion and high traction power. Gyroscopic precession is based on the below given equation [20].

$\mathrm{T}=\mathrm{J} \omega \Omega$

Where $\quad J-$ Polar moment of inertia about the axes of spin

$\omega$ - Angular velocity of the wheel

$\Omega$ - Precession rate of the wheel perpendicular to the axes of spin

Gyrover I has an external diameter of $29 \mathrm{~cm}$ and weighs about $2.0 \mathrm{~kg}$. It can achieve a speed of about $10 \mathrm{~km} / \mathrm{hr}$ and can even transverse a $45^{\circ}$ inclined ramp [20]. After Gyrover I, more developed prototypes were built, which eliminated the shortcomings of Gyrover I.

\subsection{Acrobat Mechanism}

Orientable wheels that are off centered are known as caster wheels. Caster wheels can rotate about a vertical axis which does not pass through the wheel center. The problem with robots utilizing caster wheel mechanism is that redundant actuation occurs. It is because of the fact that four actuators are needed for a robot to move on the plane surface with 3DOF. Most other mobile robots, effectively exhibit omnidirectional motion only under favorable conditions, which often are laboratory conditions. These mechanisms might not have good step climbing capability or high load carrying capacity. All these shortcomings are effectively overcome in ACROBAT mechanism. A newly developed dual-ball transmission is used for avoiding redundant actuation of the robot and a complex control system. ACROBAT stands for Active Caster Robotic drive with Ball Transmission [22]. 


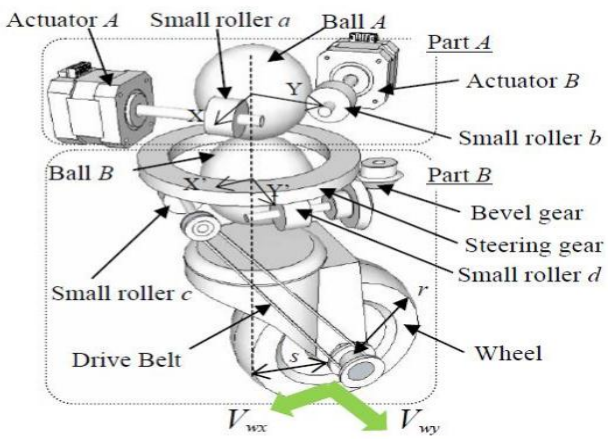

Figure 12. ACROBAT configuration [23]

The driving mechanism of the ACROBAT mechanism falls under direct-driving method. Its driving mechanism can be divided into two parts. The first part consists of two actuators with roller attachments and a driven ball $\mathrm{A}$. The two rollers rotate about their horizontal axes so that the driven ball $\mathrm{A}$ also rotates about horizontal axes. The angle between these two rollers can be changed. The second part consists of another driven ball $\mathrm{B}$ which is in contact with the driven ball $\mathrm{A}$. There are again two actuators with roller attachments in contact with the driven ball B. Due to the rotation of the driven ball A, driven ball B also rotates and its motion can be distributed through the two rollers attached to it. The rollers are orthogonal to each other. The motion control is achieved by constant kinematics control. An omnidirectional configuration robot is developed with four ACROBAT wheels in which two are passive wheels. This robot requires three motors, which remove redundant actuation. Tests were carried out to check the motional accuracy of the ACROBAT mechanism. It has an error of $10 \mathrm{~mm}$ while traveling along a length of $350 \mathrm{~mm}$ In the $\mathrm{X}$ or $\mathrm{Y}$ axis. It also records an error of $50 \mathrm{~mm}$ between the initial and final position after a rotation about $360^{\circ}$.

One novel feature of this robot is that unlike conventional ball robots, driven balls in ACROBAT mechanism have no direct contact with the ground surface. The advantage is that the traction power can be changed by controlling the contact pressure between the two balls [23].

\subsection{Omniqiu}

It is a spherical robot capable of omnidirectional mobility with holonomic capability. This robot falls under the category of direct driving method. The working principle is similar to that of a traditional ball mouse. Only that here, the working is inverted. The track ball or the driven ball in this case is driven by two orthogonally mounted rollers. The motions of the two rollers are combined to roll the driven ball, which in turn, makes the robot to move in the desired direction [24].

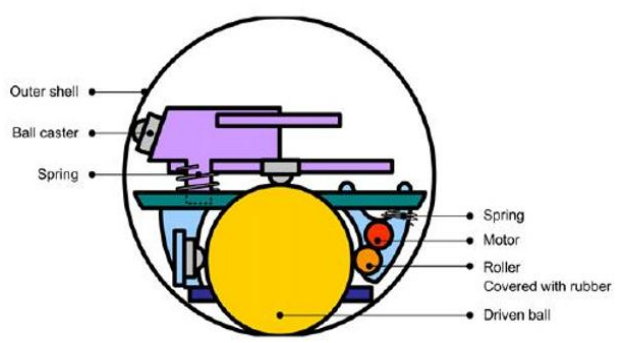

Figure 13. Structure of OmniQiu [24]

The full structure of OmniQiu can be dissected into three parts. It consists of a Main body, Driven ball and an Outer shell. The main body consists of the two rollers which drive the driven ball and all other installments inside the spherical structure except for the driven ball. The rollers are orthogonal to each other. All the components of the robot are enclosed within a spherical shell known as the outer shell. The diameter of the outer shell is $243 \mathrm{~mm}$. 


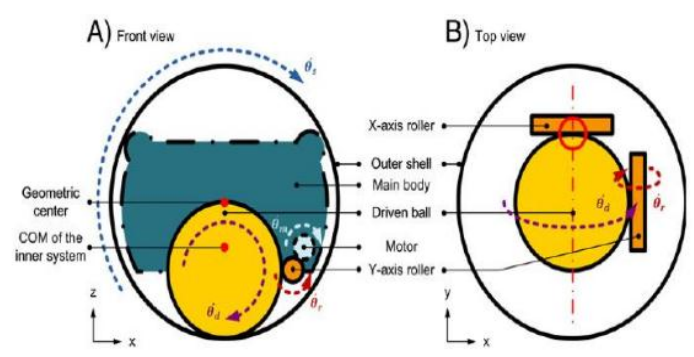

Figure 14. Driving mechanism of the OmniQiu [24]

The total mass of the robot is about $1.6 \mathrm{~kg}$. Two motors are used to drive the robot. Experimental results show that at a speed of 10,000 rpm, OmniQiu is able to achieve a forward velocity of $0.13 \mathrm{~m} / \mathrm{s}$. When the robot moves along a straight-line path, the lateral error is only $0.041 \mathrm{~m}$ for a $1 \mathrm{~m}$ length of travel. From these results, we can say that this robot is a spherical, omnidirectional robot with high motion accuracy [24].

\section{RESULTS}

On the basis of data obtained for the considered robots, relevant parameters for the field of study in the current topic are selected.All the mobile robots taken for study are compared with each other based on these parameters.

Table 1. Comparison of various robot configurations

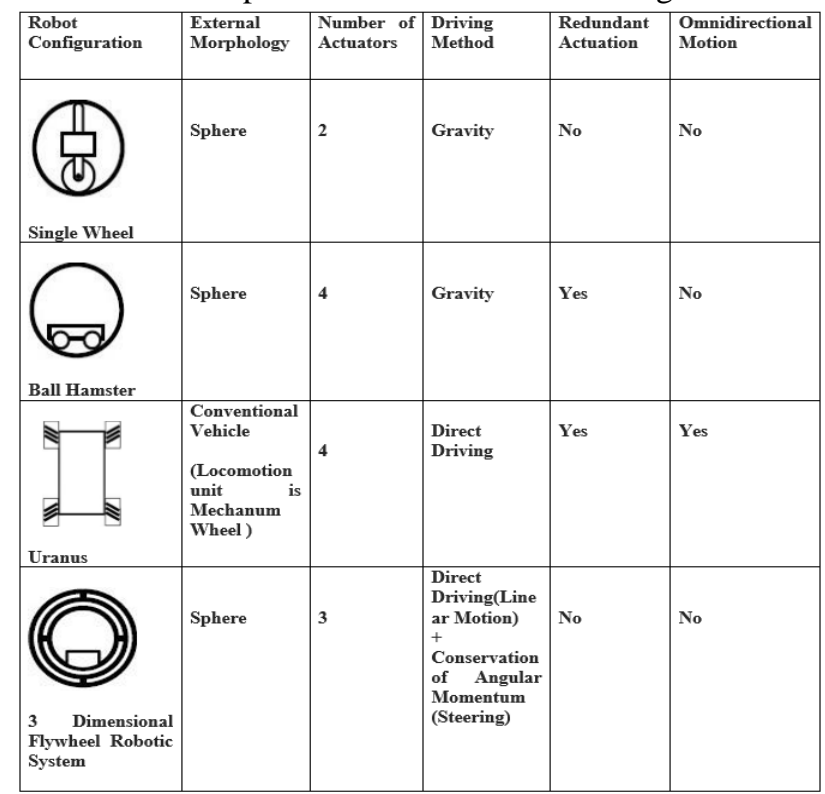



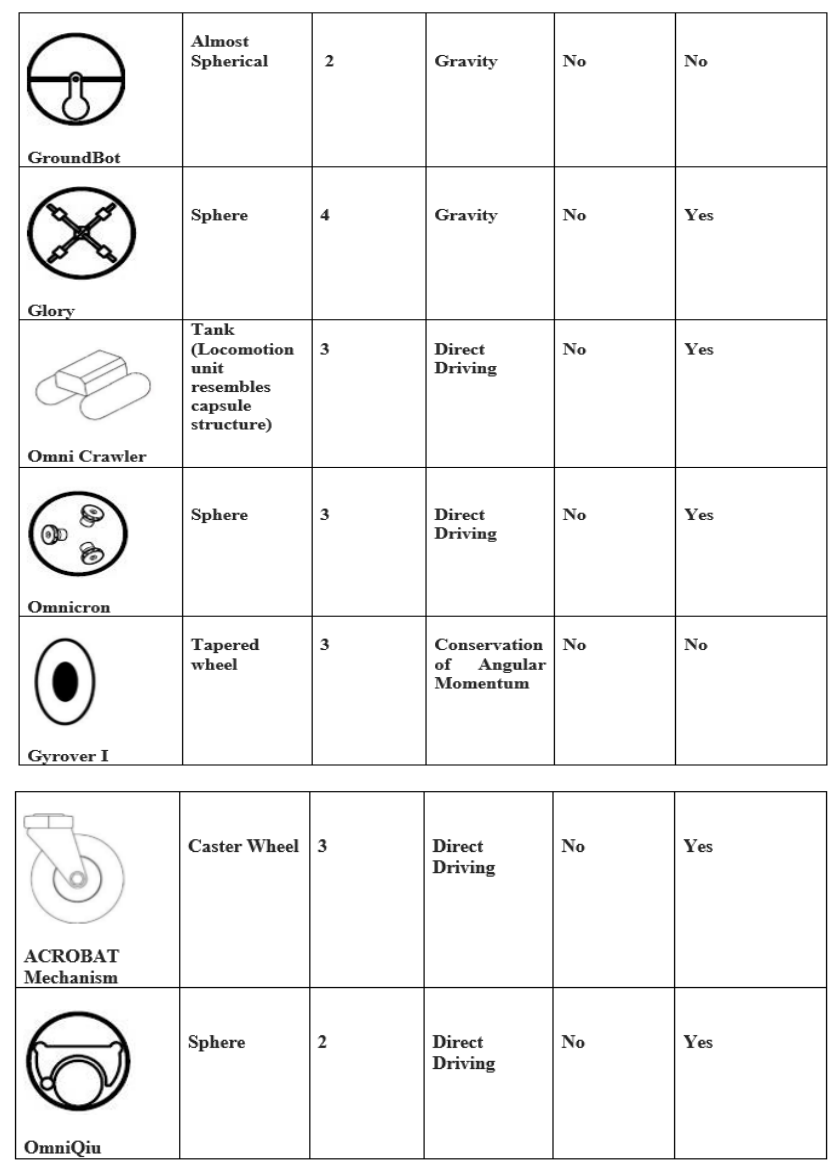

\section{CONCLUSION}

Omnidirectional robots are superior to other conventional robots in many ways. Because of their ability to move in any direction at any instant, omnidirectional robots are able to navigate their way through any obstacles without any difficulty. From the robots we have taken into consideration for studies, some conclusions can be obtained from the comparison of these robots. From Table 1, we come to understand that spherical robots need not exhibit omnidirectional motion. We know that spherical robots have geometrical symmetry and therefore it is easier for them to have omnidirectional and holonomic mobility. Out of the seven spherical robots, we have considered, only three are able to exhibit omnidirectional mobility. Also we have compared four non spherical robots, out of which three are able to exhibit omnidirectional mobility. So, we can understand that omnidirectional mobility can also be achieved by non-spherical robots. Finally, we understand that omnidirectional motion can be achieved by spherical as well as nonspherical robot. So, it is not the shape of the robot, but the driving mechanism of the robot, which determines the omnidirectional motion of the robot.

\section{REFERENCES}

[1] Robot. (2015). In Encyclopædia Britannica. Retrieved from http://www.britannica.com/technology/robottechnology

[2] Halme, A., Schönberg, T., \& Wang, Y. (1996, March). Motion control of a spherical mobile robot. Advanced Motion Control, 1996. AMC'96-MIE. Proceedings., 1996 4th International Workshop on, 1, 259-264.

[3] Alves, J., \& Dias, J. (2003). Design and control of a spherical mobile robot. Proceedings of the Institution of Mechanical Engineers, Part I: Journal of Systems and Control Engineering, 217(6), 457-467.

[4] Chen, W. H., Chen, C. P., Tsai, J. S., Yang, J., \& Lin, P. C. (2013). Design and implementation of a ball-driven omnidirectional spherical robot. Mechanism and Machine Theory, 68, 35-48.

[5] Sobh, T., Xiong, X. (2012). Prototyping of Robotic Systems: Applications of Design and Implementation: Applications of Design and Implementation.United States of America: IGI Global.

[6] Holonomic (robotics). (2015, April 14). In Wikipedia, The Free Encyclopedia. Retrieved September 19, 2015, from https://en.wikipedia.org/w/index.php?title=Holonomic_(robotics)\&oldid=656496396 
[7] Chase, R., \& Pandya, A. (2012). A review of active mechanical driving principles of spherical robots. Robotics, 1(1),3-23.(2002, January 19). Uranus [Photograph]. Retrieved from http://www.cs.cmu.edu/afs/cs/user/gwp/www/robots/Uranus.jpg

[8] Podnar,G., The URANUS Mobile Robot, Annual Report, technical report CMU-RI-TR-86-04, Robotics Institute, Carnegie Mellon University, February 1985, pg.127-129. Retrieved from http://www.cs.cmu.edu/ gwp/papers/Podnar_URANUS_1085.pdf

[9] Blackwell, M., The URANUS Mobile Robot, Carnegie Mellon University Technical Report, CMU-RI-TR-91-06, $\begin{array}{llll}\text { September } & 1990 . & \text { Retrieved } & \text { from }\end{array}$ http://www.ri.cmu.edu/pub_files/pub3/blackwell_mike_1990_1/blackwell_mike_1990_1.pdf

[10] Hsiao, C. C., Tsai, C. E., Tu, J. Y., \& Ting, Y. K. (2015). Development of a ThreeDimensional-Flywheel Robotic System. International Journal of Mechanical, Aerospace, Industrial, Mechatronic and Manufacturing Engineering, 9(4), 544-549. (2008, November 11). Rotundus [Photograph]. Retrieved from http://www.rotundus.se/_gfx/robot2_jpg.jpg

[11] Binns., Corey. (2008, September). GO TO MARS, HAVE A BALL!. Popular Science,273(3), 28-29. Retrieved from https://books.google.co.in/books?id=LTwEAAAAMBAJ\&pg=PA26\&lpg=PA26\&dq=G $\mathrm{O}+\mathrm{TO}+\mathrm{MARS},+\mathrm{HAVE}+\mathrm{A}+\mathrm{BALL}+$ popular+science\&source=bl\&ots=K2MGcvyss\&sig=XN1CslYKEMdElUk3M TsZCs2DPA\&hl=en\&sa=X\&ved=0CCMQ6AEwAmoVChMIravpi9aDyAIVRQiOCh2hQfN\#v=onepage\&q=GO \%20TO\%20MARS\%2C\%20HAVE\%20A\%20BALL\%20popula r\%20science\&f=false

[12] Kaznov, V., Backe, J.(2008). rotundus. Retrieved from http://www.rotundus.se/performance_3.html

[13] Mojabi, P. (2002). Introducing August: a novel strategy for an omnidirectional spherical rolling robot. Robotics and Automation, 2002. Proceedings. ICRA'02. IEEE International Conference on, 4, 3527-3533.

[14] Mojabi, P. (2004). Introducing glory: A novel strategy for an omnidirectional spherical rolling robot. Journal of Dynamic Systems, Measurement, and Control, 126(3), 678-683.

[15] Tadakuma, K., Tadakuma, R., Nagatani, K., Yoshida, K., \& Iagnemma, K. (2008, September). Crawler mechanism with circular section to realize a sideling motion. Proceeding of IEEE/RSJ International Conference on Intelligent Robots and Systems Acropolis Convention Center, 2422-2428.

[16] Chen, W. H., Chen, C. P., Yu, W. S., Lin, C. H., \& Lin, P. C. (2012, July). Design and implementation of an omnidirectional spherical robot Omnicron. Advanced Intelligent Mechatronics (AIM), 2012 IEEE/ASME International Conference on, 719-724.

[17] Xu, Y., Brown, H. B., \& Au, K. W. (1999). Dynamic mobility with single-wheel configuration. The international journal of Robotics Research, 18(7), 728-738.

[18] Brown Jr, H. B., \& Xu, Y. (1996, April). A single-wheel, gyroscopically stabilized robot. Robotics and Automation, 1996. Proceedings., 1996 IEEE International Conference on, 4, 3658-3663. (1996, February, 20). first gyrover [Photograph]. Retrieved from http://www.cs.cmu.edu/afs/cs/project/space/www/gyrover/first_gyrover.gif

[19] Wada, M., Hirama, T., \& Inoue, Y. (2013, July). Traction analysis for Active-caster omnidirectional Robotic drive with a Ball Transmission (ACROBAT). Advanced Intelligent Mechatronics (AIM), 2013 IEEE/ASME International Conference on, 274279.

[20] Inoue, Y., Hirama, T., \& Wada, M. (2013, November). Design of omnidirectional mobile robots with ACROBAT wheel mechanisms. Intelligent Robots and Systems (IROS), 2013 IEEE/RSJ International Conference on, 48524859.

[21] Chen, W. H., Chen, C. P., Tsai, J. S., Yang, J., \& Lin, P. C. (2013). Design and implementation of a ball-driven omnidirectional spherical robot. Mechanism and Machine Theory, 68, 35-48. 